Trinity University

Digital Commons @ Trinity

Psychology Faculty Research

Psychology Department

1989

\title{
Adult Age Differences in Knowledge of Retrieval Processes
}

L. J. Anooshian

S. L. Mammarella

Paula T. Hertel

Trinity University, phertel@trinity.edu

Follow this and additional works at: https://digitalcommons.trinity.edu/psych_faculty

Part of the Psychology Commons

Publication Details

International Journal of Aging and Human Development

\section{Repository Citation}

Anooshian, L.J., Mammarella, S.L., \& Hertel, P.T. (1989). Adult age differences in knowledge of retrieval processes. International Journal of Aging and Human Development, 29(1), 39-52. doi: 10.2190/ ERPX-9NU8-HNRM-QXTR

This Article is brought to you for free and open access by the Psychology Department at Digital Commons @ Trinity. It has been accepted for inclusion in Psychology Faculty Research by an authorized administrator of Digital Commons@ Trinity. For more information, please contact jcostanz@trinity.edu. 


\title{
ADULT AGE DIFFERENCES IN KNOWLEDGE OF RETRIEVAL PROCESSES
}

\author{
LINDA J. ANOOSHIAN \\ SUSAN L. MAMMARELLA \\ PAULA T. HERTEL \\ Trinity University
}

\begin{abstract}
We assessed knowledge of retrieval processes in young (25-35 years) and old adults (70-85 years). Both feeling-of-knowing judgments and retrieval monitoring were examined with a set of questions about recent news events. For answers that participants initially failed to recall, they rated their feeling-of-knowing as well as made predictions regarding the likelihood of recalling the answer with the aid of a specified type of retrieval cue (retrieval monitoring). Accuracy was evaluated in the context of later recall or recognition performance. We found age group differences in the accuracy of retrieval monitoring, free reciall, and recall aided by phonological cues. Using a separate inventory, we found no evidence for age group differenees in participants' knowledge of general retrieval principles.
\end{abstract}

Older adults are often acutely aware of their own memory failures [1]. Do they also know that remembering is not just a function of personal ability-that it is affected by the availability of effective retrieval cues"? To answer this question. we examined metamemorial processes of the aged. with a specific focus on knowledge of retrieval. Metamemory, generally defined as one's knowledge and beliefs about memory $[2,3]$, could relate to retrieval processes in a variety of ways. For example. knowing something about the likelihood of recalling information in specific retrieval situations should be related to the likelihood of implementing effortful retrieval strategies. With this focus as a guide, we measured the accuracy of predictions about the likelihood of retrieving specific information from memory (retrieval monitoring). as well as awareness of general retrieval principles, in young and old adults.

(C) 1989, Baywood Publishing Co.. Inc. 
Not only do good retrieval cues facilitate memory, but they seem to be especially important for older adults $[4,5]$. Of course, sophisticated retrieval strategies and abilities are unnecessary for effective remembering if good retrieval cues are readily available in the external environment. When such cues are not available, older adults appear to have difficulty. For example, long-term. memory tasks that require effortful retrieval (e.g., recall tasks) are much more sensitive to age differences than are recognition tasks [6]. In general, older persons appear to be at a disadvantage when successful retrieval requires self-generated, reconstructive procedures; they may be less adept at using elaborate retrieval mechanisms [6] and/or may require a longer time [7] or greater effort [8] for memory search. Retrieval processes have frequently been implicated in previous studies of adult age group differences in memory.

We examined participants' monitoring of both the availability of specific information in memory and its accessibility with the aid of particular types of retrieval cues. For this purpose, we constructed the News-Knowledge Questionnaire by composing questions about significant political events that had occurred in the last five years. For items that participants failed to answer correctly through free recall, we examined the accuracy of both feeling-ofknowing judgments and predictions about later retrieval with specified types of cues. When accuracy is assessed in the context of participants predictions for recognition, it reflects the accuracy of feeling-of-knowing judgments $[9,10]$; high confidence ratings should be followed by correct recognition and low ratings followed by recognition failures. In contrast, retrieval monitoring reffects the accuracy of predicting the likelihood of retrieving the correct answer when provided with cues (in this case, either related information about the news event or phonological cues). In light of adult age differences in retrieval, the present study is most significant in differentiating between feeling-of-knowing, as defined and examined by past researchers [9-14], and the new concept of retrieval monitoring.

Researchers have examined feeling-of-knowing judgments with the hope of providing a better understanding of the retrieval processes of elderly persons. Lachman et al. suggested that accurate feeling-of-knowing judgments were especially important to elderly adults when their attempts at direct retrieval failed [13]. That is, elderły adults depend on accurate metamemory to guide processing associated with further search or inferences from related information. Consistent with previous results obtained by Perlmutter [14], Lachman et al. found no evidence of an age-related decline in the accuracy of feeling-ofknowing judgments. However, in designing the present study. we saw the need for further assessments of the fecling-of-knowing judgments of elderly adults. Examination of Lachman et al.'s complete questionnaire indicated that the items fell into several broad categories-e.g. geography, mytiology, literature [13]. It seemed that respondents could do fairly well by identifying which of the broad content areas were most familiar to them rather than by generating a 
feeling-of-knowing judgment for each particular answer. In contrast, all items in our questionnaire were from a similar content domain-political events with special news significance. Hence, accurate feeling-of-knowing judgments required that participants be able to judge how much they knew about particular events rather than simply being able to differentiate between familiar vs. unfamiliar domains of knowledge.

For the present study, it seemed unwise to focus exclusively on feeling-ofknowing tasks which relied on recognition performances for assessments of metamemorial accuracy [cf. 10, 13, 14]. Should one reasonably expect to find metamemorial deficits in the context of memory tasks with which older adults have little difficulty (i.e., recognition tasks)? Further, the hypothesis that metamemory serves a compensatory function for elderly adults [13] seems to depend on the demonstration of good retrieval monitoring in addition to accurate feeling-of-knowing judgments. If they know little about the consequences of potential retrieval cues, it seems unlikely that older adults would generate or search for related information (cues) to compensate for initial recall failures. In our tasks, good retrieval monitoring depends on more sophisticated knowledge than simply whether information is available in memory; a respondent must decide whether information, when available in memory, can be accessed with the aid of a particular type of retrieval cue.

Finally, we reasoned that there were two major components of metamemorial knowledge of retricval processing. In addition to our interest in participants' monitoring of their own processing (retrieval monitoring), we were interested in their general knowledge of retrieval principles. Explicit awareness of retrieval principles was evaluated through responses to the General Retrieval Inventory [15]. Items in this inventory reflected particular types of relationships between sought-after memories and those types of cues that, according to previous memory research, are conducive to effective retrieval. Effective cues include verbal information that is similar to the elusive memory along a phonological dimension [16, 17] or a semantic dimension [18]. Also, environmental contexts during encoding aid memory when reinstated at the time of retrieval. The room in which information was originally encoded, for example, provides effective retrieval cues $[19,20]$. If an elderly adult simply does not know that remembering depends on these different kinds of retrieval cues, it is unlikely that their construction or use would be implemented in a retrieval strategy.

\section{METHOD}

\section{Participants}

We tried to minimize the potential impact of individual and cohort variability by selecting participants of approximately the same socio-economic status and intellectual level, excluding participants who were enrolled in school or 
functioned as educators, and assessing their memory for meaningful and relatively permanent, real-world knowledge and events. The unfamiliarity and anxiety that might be caused by a laboratory setting-especially for old adultswas reduced by conducting the research within the participant's home environment.

There were two groups of twenty participants each. The young age group consisted of persons twenty-five to thirty-five years of age $(8$ males and 12 females), and the old age group consisted of participants between seventy and eighty-five years of age ( 3 males and 17 females). All lived independently in the community and were individually recruited through contacts with various community groups. The mean number of years of schooling for young adults was 15.5, and the mean for old adults was 14.0. Although young adults obtained somewhat higher scores on the vocabulary subtest of the WAIS.R ( $M=62.0$ ) than did old adults ( $M=59.3)$, the difference was not significant.

Results of a preliminary inventory administered to all participants indicated that none had experienced a major health problem or a major life experience that affected exposure to the mass media within the past five years. Questions about exposure to newspapers, radios, T.V. news, and magazines failed to provide evidence for group differences in past exposure to news events. Several potential participants who reported visual or auditory deficits were excluded from the study.

\section{Materials}

The News-Knowledge Questionnaire was composed of sixty news-related questions covering major political events during the five years preceding the starting date of this investigation. The items were of major news significance taken from the end-of-the-year issues of Time magazine, thus ensuring exposure from several different forms of media presentation. For each question, three types of cues were constructed: a piece of additional information about the news event (related-information cue), the beginning sound of the to-beremembered word (phonological), and multiple-choice options. One prototypical question was phrased as follows: "Congress has established a new legal holiday, to begin in 1985, honoring a famous American. Who is that person?" Recognition options included John F. Kennedy, Thomas Jefferson, Robert F. Kennedy, Martin Luther King, and Dwight D. Eisenhower. The related-information cue for this question was "He was a famous civil rights lcader." The phonological cue was "The person's last name begins with 'ki' (enunciated)."

The General Retricval Inventory contained five statements that addressed the effects of particular types of cues on remembering (Statements 2-6; see Table 1). Each statement was worded so that agreement would indicate participants had been explicitly aware of the benefits of a particular type of cue in their past attempts at retrieving forgotten information. Several additional 
Table 1. The General Retrieval Inventory

1. In general, I believe I have a good memory.

2. When trying to remember something, I have an easier time when I happen to be thinking about something else that is meaningfully connected to what I want to remember.

3. When trying to remember something, I have an easier time when I see or hear something else that is meaningfully connected to what I want to remember.

4. When trying to remember something, it's easier for me when I happen to be thinking about or hearing something that sounds like the word (s) that I want to remember.

5. When trying to remember something, I have better luck if I happen to be in the place I originally heard it, saw it, or found out about it.

6. When trying to remember something, I can do so more easily if there is an object present that was there when I originally heard it, saw it, or found out about it.

7. I often remember things "spontaneously."

8. When I've forgotten something, I am usually confident that the memory will come back to me at another time.

statements were designed to tap general confidence in memory. Although largely unrelated to the primary focus for this study. it seemed potentially significant that the self-reports of elderly adults frequently emphasize memory failures [1]. If they lack confidence, old adults may be less willing to commit the mental effort necessary for successful retrieval operations. doubting their ability to find the answer with that additional effort.

\section{Procedure}

Participants werc individually tested in their homes with the News-Knowledge Questionnaire, the General Retrieval Inventory, and the WAIS-R vocabulary subtest. The examiner read all questions aloud and the participant responded to them orally. Half the participants in each age group were given the General Retrieval Inventory prior to the News-Knowledge Questionnairc. The WAIS-R vocabulary subtest was always administered last.

The procedure for the News-Knowledge Questionnaire was divided into two phases. In Phase 1, the examiner assessed free recall of answers to sixty questions. For any question answered incorrectly (including no response), participants were asked to rate their feeling of knowing on a 7-point Likert scale (with endpoints labeled "Very confident that I don't know" and "Very confident that I know"). 
Participants responded by pointing to a large piece of white paper upon which the scale was printed. Immediately after this rating, they rated the likelihood of retrieving an answer if given a specific type of cue-phonological, additional related information, or multiple-choice items. (An example of each of the different cue types was given during the initial explanation of the procedure.) The endpoints of the Likert scale for the retrieval ratings were labeled "Not very likely" and "Very likely."

The order in which the different cues were presented varied across two different lists. Each of the two lists was constructed by randomly arranging the cue types within blocks of three (e.g.. phonological, related-information, multiple-choice), thus ensuring that participants had approximately equal exposure to each type of cue. The assigned order was followed for those items that participants originally failed to answer correctly. For example, if phonological was first in the assigned cue order, then. for the first question participants failed to answer correctly, they were asked to rate the likelihood of remembering if given the first sound of the answer. List assignment ( 1 or 2 ) was counterbalanced with the two orders of the inventories.

Participants had no opportunity for feedback regarding the accuracy of the feeling-of-knowing or retrieval ratings that they provided in Phase 1. During Phase 2. the examiner returned to each question missed (and rated by the subject) and provided the kind of cue that the participant had considered for retrieval ratings in Phase 1 . The responses were recorded.

For the General Retrieval Inventory, participants rated the extent to which they agreed with each of eight statements read by the examiner (from "Strongly disagree" to "Strongly agree").

\section{RESULTS}

The multivariate approach to analysis of variance was used for the analyses of each of the major dependent measures. This approach has been recommended by McCall and Appelbaum for repeated-measures designs because it avoids the assumption of homogeneity of covariances required by the univariate approach [21]. Approximate univariate $F$ s for Wilks lambda criteria are reported; the significance level was set at .05 .

\section{Feeling-of-Knowing Judgments}

The usual approach to assessing accuracy for feeling-of-knowing judgments involves examining the percentage of correct recognition choices for different rating categories [13]. Accurate judgments would be reflected in higher percentages for items that participants had originally missed but indicated they knew than for items associated with lower confidence ratings. This type of analysis was not workable for the present study. In contrast to past research 
with a single retrieval-cue situation (recognition), our method was to distribute missed items across three different retrieval-cue situations. Hence, the total number of missed items for recognition trials-broken down by confidence category -would be prohibitively small.

We used a different method for assessing metamemorial accuracy. This assessment was, of course, based only on those items that the participant failed to answer correctly in Phase 1. Feeling-of-knowing ratings were averaged for items associated with either successful or unsuccessful remembering in Phase 2. Metamemorial accuracy should be reflected in a higher mean rating (Phase 1) for items that were remembered than for items the participant failed to remember in Phase 2.

For both the young and old age groups, mean ratings for feeling-of-knowing were higher for items followed by successful remembering ( $M \mathrm{~s}=5.16$ and 4.65 , respectively) than for items followed by continued memory failure in Phase 2 $(M s=3.94$ and 3.60, respectively). A two-way ANOVA (age group X Phase 2 memory) revealed only a significant main effect for Phase 2 memory, $F(1,38)=$ 64.59. Similar means were calculated for participants' ratings of the likelihood of recognizing the correct answer from multiple-choice options. Means for recognition predictions were higher for items followed by correct than incorrect recognition in Phase 2. both for the young ( $M s=5.80$ and 3.95 , respectively) and the old age group ( $M s=5.27$ and 3.05, respectively). Consistent with the reported results for general feeling-of-knowing ratings, a two-way ANOVA (age group $\mathrm{X}$ Phase 2 recognition) on these mean scores for recognition predictions revealed only a main effect for Phase 2 recognition, $F(1,38)=28.73$.

\section{Retrieval Monitoring}

Retrieval monitoring was evaluated in the same fashion as described above for feeling-of-knowing judgments. However, the means for each participant were for the ratings provided as cued-recall predictions (i.e., the likelihood of remembering if provided with related-information or phonological cues). As can be seen in Table 2, participants consistently gave higher ratings when predicting cued recall for items that they successfully recalled in Phase 2. A three-way ANOVA (age group $X$ type of cue $X$ Phase 2 recall) yielded a significant main effect for Phase 2 recall, $F(1,38)=58.60$, reflecting the difference between ratings followed by successful vs. unsuccessful recall. This difference tended to be greater (indicating greater metamemorial accuracy) for young than old adults, as well as greater for phonological-cue than related-information items. However, the implicated interactions between Phase 2 recall and age group, $F(1,38)=$ 3.83, and between Phase 2 recall and type of cue, $F(1,38)=4.07$, were only marginally significant ( $p \mathrm{~s}=.058$ and .051 , respectively).

One difficulty with our approach to retrieval monitoring is that cued-recall predictions should not be entirely independent of feeling-of-knowing. For 
Table 2. Mean Rating and Change Scores for Cued-Recall Predictions for the Two Age Groups

\begin{tabular}{lcc}
\hline & \multicolumn{2}{c}{ Age group } \\
\cline { 2 - 3 } Retrieval situation & Young & Old \\
\hline \multicolumn{1}{c}{ Mean rating for cued-recall prediction } \\
Related-information cue & & \\
Successful recall & 5.47 & 4.89 \\
Unsuccessful recall & 4.22 & 4.02 \\
Phonological cue & & \\
Successful recall & 5.68 & 5.02 \\
Unsuccessful recall & 3.12 & 3.63 \\
& & \\
$\quad$ Mean change from feeling-of-knowing rating \\
Related-information cue & & .31 \\
$\quad$ Successful recall & .50 & .43 \\
Unsuccessful recall & .13 & .36 \\
Phonological cue & & \\
$\quad$ Successful recall & .69 & \\
Unsuccessful recall & &
\end{tabular}

example. if a participant is quite confident about not knowing something and is asked to predict cued recall, it is unlikely to matter whether the upcoming cues were to be phonological or related information. Further, it is likely that participants who used the high end of the Likert scale for one type of rating would also do so for other ratings. Indeed, the correlations between mean feeling-of-knowing ratings and mean cued-recall predictions were high and significant for each age group ( $r s>.50$ ).

Because participants provided both a feeling-of-knowing judgment and a retrieval prediction for every question missed in Phase I, we could evaluate retrieval monitoring by determining the extent or direction of change from ratings of feeling-of-knowing to ratings for cued-recall predictions. In general, participants tended to provide higher ratings for recall predictions than for feeling-of-knowing. However. our primary interest was in the extent to which this change in ratings was predictive of the participant's later success in cued recall. Hence, for both phonological and related-information items, we calculated the mean change in ratings (recall-prediction minus feeling-of-knowing rating) for items followed by either successful or unsuccessful recall in Phase 2.

As can be seen in Table 2, it was only for young adults that changes in ratings appeared to be predictive of actual cued-recall performance. These change scores 
were analyzed with a three-way ANOVA with factors for age group, type of cue. and Phase 2 recall. That analysis confirmed both a main effect for Phase 2 recall, $F(1,38)=5.62$, and a significant $P$ hase 2 recall $X$ age group interaction, $F(1,38)=5.34$. The interaction reflected that the simple main effect for Phase 2 recall (success vs. failure) was significant only for young adults. It appeared that old adults failed to make meaningful distinctions between feelingof-knowing judgments and cued-recall predictions. When the two ratings differed, that difference was unrelated to whether or not the participant's later cued-recall at tempt would be successful.

\section{Responses on the General Retrieval Inventory}

Young and old adults provided similar ratings for statements on the General Retrieval Inventory that tapped knowledge of retrieval-cue effectiveness. These ratings were analyzed using a two-way analysis of variance with factors for age group and item (Items $2-6,8$ ). This analysis revealed only a significant effect for item, $F(5,34)=4.65$. Participants agreed most with the control statement ( $\$ 8)$-that they would be likely to remember something at a later time. The ratings for this statement $(M=5.85)$ were significantly higher than ratings for statements that referred to the availability of environmental/ contextual cues, whether these cues took the form of spatial locations (Statement $5, M=4.38$ ) or physical objects (Statement 6, $M=4.53$ ). Ratings for the control statement were not significantly different from ratings for remaining statements ( $M s=5.05,5.38,5.00$ for Statements 2, 3, and 4, respectively).

Old adults tended to give lower ratings to items that addressed general memory ability than did young adults. In responding to Statement 1, old adults indicated less confidence in their memory $(M=4.60)$ than did young adults $(M=5.70)$ and were less likely to agree that they remember information spontaneously (Statement $7, \boldsymbol{M}=5.80)$ than were young adults $(\boldsymbol{M}=6.20)$. However, neither difference was significant.

\section{Memory Performances}

Our focus on retrieval monitoring was derived from past research indicating that age group differences in memory are most apparent for tasks requiring effortful retrieval processes. Consistent with this past research, young adults in this study provided more correct answers in their first exposure to the NewsKnowledge Questionnaire ( $M=37.95$ vs. 30.40 for old adults), $F(1,38)=$ 6.58. Because of poorer free recall in Phase 1. older adults had more opportunities to retrieve further correct answers in Phase 2-when specific retrieval cues were provided.

Thus, to examine cued-recall and recognition, we calculated proportion scores: the number of correct answers divided by the number of attempted items. These proportions were calculated separately for the three retrieval 
situations (phonological, related-information, multiple-choice). A two-way ANOVA with factors for age group and retrieval situation revealed a main effect for retrieval situation, $F(2,37)=33.03$. Recall with related-information cues $(M=.44)$ was significantly worse than either recall with phonological cues $(M=.69)$ or recognition $(M=.70)$. Further, the age group $\mathrm{X}$ retrieval situation interaction was significant, $F(2,37)=3.68$. It was only for recall with phonological cues that young adults ( $M=.79$ ) significantly outperformed the old adults $(M=.61)$. Mean proportion scores for the young and old adults were quite comparable for recall with related-information cues ( $M s=.47$ and .41 . respectively) and for recognition ( $M s=.68$ and .71 , respectively). Overall, these findings are consistent with the argument that age group differences in free recall reflect differences specific to retrieval processing rather than generalized memory deficits for elderly persons.

\section{DISCUSSION}

Our results indicated age group differences in retrieval processing as well as corresponding age group differences in retrieval monitoring. Age group differences in memory for news events were significant for free recall and for recall with phonological cues, but not for recall cued by related information or for the recognition of answers to specific questions about recent news events. Although the group difference for recall with phonological cues was unexpected. our results were generally consistent with past research in indicating that specific answers were equally available to participants in the two age groups-that group differences in memory reflected differences in retrieval processes. The major goal of the present research was to identify new measures of metamemory-as yet unexplored in previous research-that could be helpful in understanding the retricval processing of older adults. Toward this goal, our results pointed to the significance of retricval monitoring as a promising new concept for further examination.

We designed our News-Knowledge Questionnaire such that feeling-of-knowing judgments required distinctions among items within the same general content area (political events). Despite the apparent difficulty of this task, our results were quite comparable to those reported previously [13]. Participants in both age groups made accurate feeling-of-knowing judgments. as well as accurate predictions about recognition performance. Yet, for cued-recall predictions, the results confirmed the hypothesized age group differences in monitoring the likelihood of recall with phonological or related-information cues. For older adults, the accuracy of cued-recall predictions appeared to reflect only the accuracy of fecling-of-knowing ratings. That is, differences between the two ratings appeared unrelated to the actual effectiveness of the cue that the participant had considered for cued-recall predictions. These results reinforce the need for broader theoretical and empirical treatments of the concept of 
memory monitoring than has typified much past research $[9,11-14]$. Knowing whether information can be retrieved (feeling-of-knowing) may be less important for effective retrieval processing than one's monitoring of the effectiveness of particular types of cues.

Of course, definitive conclusions regarding relationships between retrieval processing and retrieval monitoring must await further experimental study. Our results can, nevertheless, be useful in evaluating the plausibility of specific relationships hypothesized by others. In this regard, our results were not consistent with the compensatory relationship between metamemory and memory, as had been proposed by Lachman et al. [13] . Even though accurate in feeling-of-knowing judgments, the observed retrieval-monitoring abilities of older adults seem inconsistent with the suggestion that older adults compensate for retrieval deficits by depending on metamemory to guide further search and inference attempts. Further, the notion of compensatory relationships appears to contradict other evidence that memory difficulties for older adults tend to be restricted to tasks that require effortful processing [6]; compensatory processes. Macht and Buschke have obtained evidence indicating that elderly persons require a greater proportion of their limited processing capacity for retrieval tasks (e.g., search, recovery, and decision operations) than do young adults [8].

We suggest that poor retrieval monitoring may contribute to the elderly adult's inefficient use of a limited processing capacity. Obviously, free recall will suffer if one relies only on attempts at direct retrieval and fails to search and/or generate the most appropriate cues for retrieval. If the effectiveness of a type of cue is overestimated, one may divert a significant portion of the processing capacity to unproductive search and retrieval attempts. Older adults may require more effort for retrieval because their limited processing efforts are managed unwisely or inefficiently; if so, retrieval monitoring seems a likely culpit.

In addition to a broader perspective for research on memory monitoring-a perspective that includes both feeling-of-knowing judgments and retrieval monitoring-greater breadth is needed in further studies of retrieval monitoring. Our results with related-information cues emphasized the need for future research with different types of retrieval-cue situations. Cued-recall scores were significantly lower for related-information than for phonological cues, indicating that many of our related-information cues were simply ineffective in aiding retrieval. What an experimenter defines as "related information" will only be effective in aiding recall if, of all the many possibilities, respondents were lucky enough to get a cue that was meaningful in the context of other things they happened to know about the news event. We recommend that future researchers examine retrieval monitoring by providing participants with more specific knowledge about the nature (e.g., the content domain) of upcoming relatedinformation cues. For example, subjects could predict the likelihood of 
remembering if told the geographical area where an event occurred or the names of other individuals associated with the news event.

To achieve a fuller understanding of the retrieval processing of elderly adults, it is likely that research examining participants' monitoring of their own retrieval processing will be more fruitful than research examining their knowledge of general retrieval principles. No significant age group differences were found for items from the General Retrieval Inventory. Nevertheless, it may be important that our participants-regardless of age-seemed to be unaware that environmental/contextual cues could serve as effective retrieval cues for remembering. Anooshian et al. have reported that. for college students who were new to the college campus (i.e.. were in an unfamiliar retrieval context). knowledge of the benefits of environmental cues was positively related to both self-esteem and assertiveness [15]. It seemed that students with poor metamemory mistakenly attributed their memory failures to their own cognitive deficiencies rather than to the loss of effective retrieval cues. Similarly, it may be that, without such knowledge of the effectiveness of environmental cues, elderly adults who relocate-often after many years of living in a highly familiar environment-may be especially vulnerable to incorrect attributions that memory failures reflect their own inadequacies; such misattributions would be reinforeed by mistaken notions of the generalized and inevitable memory decline associated with aging. Hence, while knowledge of retrieval principles may not be useful for understanding retrieval processing. assessments of such knowledge may take on more significance in the context of the low self-esteem and depression often expressed by elderly adults [22].

\section{ACKNOWLEDGMENTS}

We exiend our thanks to Charles B. White for guidance in selecting and screening participants for the study and to Mary Dozier for helpful comments on earlier drafts of the manuscript.

\section{REFERENCES}

1. J. C. Cavanaugh, J. G. Grady, and M. Perlmutter, Forgetting and the Use of Memory Aids in 20 to 70 Year Old's Everyday Life, International Journal of Aging and Human Development, 1721), pp. 113-122, 1983.

2. A. L. Brown, The Development of Memory: Knowing, Knowing about Knowing, and Knowing How to Know, in Advances in Child Development and Behavior. Vol. 10, H. W. Reese (ed.), Academic I'ress. New York, pp. 103-152,1975.

3. A. L. Brown, Knowing When, Where, and How to Remember: $\wedge$ l'roblem of Metacognition, in Advances in Instructional Psychology, Vol. I. R. Glaser (ed.), Lawrence Erlbaum Associates, Hillsdale, New Jersey, pp. 77-165, 1978 . 
4. D. F. Hultsch, Adult Age Differences in Retrieval: Trace-dependent and Cue-dependent Forgetting, Developmental Psychology, 11, pp. 197-201, 1975 .

5. H. A. Taub, Aging and Free Recall, Journal of Gerontologv, 23, pp. 466$468,1968$.

6. F. M. Craik, Age Differences in Human Memory, in Handbook of the Psychology of Aging, J. E. Birren and K. W. Schaie (eds.), Van Nostrand Reinhold Co., New York, pp. 384-420, 1977.

7. D. J. Madden, Age-related Slowing in the Retrieval of 1 nformation from Long-term Memory, Journal of Gerontology, 40, pp. 208-210, 1985.

8. M. L. Macht and H. Buschke, Age Differences in Cognitive Effort in Recall, Journal of Gerontology, 38, pp. 695-700, 1983.

9. J. T. Hart, Memory and the Feeling-of-knowing Experience, Journal of Educational Psychology, 56, pp. 208-216, 1965.

10. R. Lachman and J. L. Lachman, Age and the Actualization of World Knowledge, in New Directions in Memory and Aging, L. W. Poon, J. L. Fozard, L. S. Cermak, D. Arenberg, and L. W. Thompson (eds.), Lawrence Erlbaum Associates, Hillsdale, New Jersey, pp. 519-544, 1980.

11. M. Gruneberg and J. Monks, Feeling of Knowing and Cued Recall, Acta Psychologica, 38, pp. 257-265, 1974.

12. J. T. Hart, Memory and the Memory Monitoring Process, Journal of Verbal Learning and Verbal Behavior, 6, pp. 685-691, 1967.

13. J. L. Lachman, R. Lachman, and C. Thronesbery, Metamemory through the Adult Life Span, Developmental Psychology, 15(5), pp. 543-551, 1979.

14. M. Perlmutter, What is Memory Aging the Aging of? Developmental Psychology, 14, pp. 330-345, 1978.

15. L. J. Anooshian, P. Ashbrook, and P. T. Hertel, Self-esteem and Beliefs about Memory in Environmentally Stable and Relocated Students. Journal of Research in Personality, 19, pp. 457-471, 1985.

16. J. D. Bransford, J. J. Franks, C. D. Morris, and B. S. Stein, Some Ceneral Constraints on Learning and Memory Research, in Levels of Processing and Human Memory, L. S. Cermak and F. I. M. Craik (eds.), Lawrence Erlbaum Associates, Hillsdale, New Jersey, 1979.

17. D. L. Nelson, J. W. Wheeler, R. C. Borden, and D. H. Brooks, Levels of Processing and Cueing: Sensory vs. Meaning leatures, Jourmal of Experimental Psychology, 103,pp.971-977, 1974.

18. D. M. Thomson and E. Tulving, Associative Encoding and Retrieval: Weak and Strong Cues, Journal of Experimental Psycholog.', 86, pp. 255-262. 1970.

19. P. T. Hertel, L. J. Anooshian, and P. Ashbrook, The Accuracy of Beliefs about the Benefits of Retrieval ('ues, Memory and C'ognition. 14, pp. 265$269,1986$.

20. S. M. Smith, Enhancement of Recall using Multiple Environmental Contexts during Learning, Memory and Cognition, 10, pp. 405-412, 1982.

21. R. B. McCall and M. 1. Appelbaum, Bias in the Analysis of Repeatedmeasures Designs: Some Alternative Approaches, Child Development, 44, pp. $401-415,1973$. 
22. N. Butters, Potential Contributions of Neuropsychology to Our Understanding of the Memory Capacities of the Elderly, in New Directions in Memory and Aging, L. W. Poon, J. L. Fozard, L. S. Cermak, D. Arenberg, and L. W. Thompson (eds.), Lawrence Erlbaum Associates, Hillsdale, New Jersey, pp. 451-459, 1980.

Direct reprint requests to:

Linda J. Anooshian

Department of Psychology

Boise State University

Boise, 1D 83725 